\title{
Autorisation à pratiquer les activités cliniques et biologiques d'assistance médicale à la procréation
}

Définies aux articles L. 184-1, L. 673-5 et L. $712-2\left(2^{\circ}, b\right)$ du Code de la Santé Publique

Une première liste (J.O. du 6 Août 1996) a été publiée dans Andrologie, V. 6 n $^{\circ} 3$, Septembre 1996, 332-353.

Nous présentons ici une mise à jour de cette liste complétée à partir des J.O. du 14 mars 1997 (p.4051) du 12 avril (p.5582-5585) et du 16 avril (p.5748-5751).

\section{Activités cliniques}

1- Recueil par ponction d'ovocytes pour AMP intraconjugale

2- Transfert d'embryons

3- Recueil par ponction d'ovocytes en vue de dons

4- Recueil par ponction de spermatozoïdes 
- Centre médico-chirurgical de Schiltigheim

M. Dellenbach, Mmes Moreau et Ohl $(1,2)$

- Fondation du diaconat, Mulhouse

Mme Lamarca-Roth $(1,2)$

\section{REGION AQUITAINE}

- CHU de Bordeaux

MM. Dallay, Horovitz, Hocke et Leng (1,2,3), Pariente (4)

- Polyclinique de Bordeaux-Nord-Aquitaine

M. Etchevery (4)

- Clinique Saint Sernin, Bordeaux

M. Audebert $(1,2,4)$

- Clinique Larfague, Bayonne

MM. Molia, Briend et Mme Haran $(1,2)$

- Clinique Lagrange, Pau

MM.Acharian et Rose $(1,2)$

- Polyclinique Francheville, Périgueux

MM.Girard, Hourcarie et Malauzat $(1,2)$

\section{REGION AUVERGNE}

- CHU (Hôtel-Dieu) de Clermont-Ferrand

M. Pouly $(1,2,3,4)$, M. Canis $(1,2,3), M$. Hermabessière (4)

- Clinique La Pergola, Vichy

M. Manhes $(1,2)$

\section{REGION BOURGOGNE}

- Clinique médico-chirugicale de Chenôve (Côte d'Or)

M. Halfon $(1,2)$

- CHR de Dijon

M. Sagot $(1,2,3)$

\section{REGION BRETAGNE}

- CHR de Rennes

MM. Giraud, Poulain, Grall et Mme Laurent $(1,2,3)$ MM. Lobel et Staerman (4)

\section{- Clinique Mutualiste La Sagesse, Rennes}

MM. Rio, Bouchet, Brow, Priou et Vialard $(1,2,3)$ M. Leveque (4)

- CHRU du Morvan à Brest

Mme Le Martelot (1,2,3,4), M.Collet (1,2,3), M.Fournier (4)

- Clinique Pasteur, Brest

Mmes Chabaud et Hassoun $(1,2)$

\section{REGION CENTRE}

- CHR de Lorient

Mme Julou $(1,2)$ 
- CHR de Dreux

M. Tribalat $(1,2)$

- Clinique du Parc, Chambray-les-Tours

MM. Gallier et Forveille (1,2), M. Vannier (4)

- CHU de Tours

M. Lansac, $(1,2,3,4)$, Mme Clouet $(1,2,3)$

\section{REGION CHAMPAGNE-ARDENNE}

- CHR de Charleville-Mézières

M. Mereb (1)

- Polyclinique de Courlancy, Reims

MM. Giacomini et Chaste (1,2), M. Amory (4)

- CHU de Reims

M. Gabriel (1,2,3,4), MM. Harika et Quereux $(1,2,3)$, M. Lardennois (4)

\section{REGION FRANCHE-COMTE}

- CHU de Besançon

M. Agnani $(1,2,3)$, M. Bittard (4)

- Polyclinique du Centre Les Cigognes, Besançon

MM. Guedj et Lelouch $(1,2)$

\section{REGION ILE DE FRANCE}

\section{- Hôpital Necker-Enfants malades, Paris 15}

MM. Dufour et Vogt (4)

- Hôpital Cochin, Paris 14

MM. Zorn et Douard (1,2,3,4), M. Thiounn (4)

- Hôpital Saint Vincent de Paul, Paris 14

Mme Epelboin $(1,2,3)$, Mme De Mederos $(1,2)$

- Groupe Hospitalier La Pitié-Salpétrière, Paris 13

Mme Vauthier-Brouzes et M.Lefebvre (1,2), Haertig (4)

- Hôpital Tenon, Paris 20

MM. Salat-Baroux et Antoine (1,2,3,4), M. Merviel (1,2,4), MM. Arvis, Tritto et Bittard (4)

- Hôpital Antoine Beclère, Clamart (Hauts de Seine)

M. Frydmann $(1,2,3)$ et M. Olivennes $(1,2,3,4)$

- Hôpital Jean Verdier, Bondy (Seine Saint Denis)

Mme Uzan (1,2,3), M. Sellam (4)

- Hôpital Bicêtre, Le Kremlin-Bicêtre (Val de Marne)

MM. Jardin, Benoit et Izard (4)

- Hôpital Bichat, Paris 18

MM. Madelena et Beniflat $(1,2)$

- Hôpital des Métallurgistes, Paris 11

Mmes Bulwa et Hatem (1,2)

- Hôpital des Diaconesses, Paris 12

M. Larue $(1,2)$ 
- Clinique Spontini, Paris 16

Mme Menard, MM.Behnamou et Fauck $(1,2)$

- Institut Mutualiste Montsouris, Paris 14

M. Sarot $(1,2,3,4)$

- Clinique de la Muette, Paris 16

Mme Alvarez, MM.Chouraqui, Debache et Cornier $(1,2)$, Cohen et Hazout $(1,2,4)$

- Centre Médico-chirurgical Parly II, Le Chesnay (Yvelines)

M. Kerbrat $(1,2)$

- Clinique Sully, Maisons Lafitte (Yvelines)

M. Naert $(1,2)$

-Centre Hospitalier inter communal de Poissy (Yvelines)

M. Bailly $(1,2,3,4)$, MM. Nisand, Wainer et Philippe $(1,2,3)$

- Clinique du Blanc Mesnil (Seine Saint-Denis)

MM. Dujardin, Laffy et Nataf $(1,2)$

- Clinique de la Dhuys, Bagnolet (Seine Saint Denis)

MM. Berthelot,Cornet, Cukierman, Ayel et Raveneau (1,2) M. Amar (4)

- Centre chirurgical Pierre Cherest, Neuilly (Hauts de Seine)

Mmes Nathan et Glissant et MM. Laffy, Aubriot (1,2) MM. S et M. Adjiman (4)

- Hôpital américain de Paris, Neuilly (Hauts de Seine)

M. Thebault (1,2), M. Chiche (4)

- Clinique de la Roseraie, Aubervilliers (Seine Saint Denis)

M. Allart (1,2), MM. Braun et Rozan (4)

- Centre hospitalier intercommunal Jean-Rostand, Sèvres (Hauts de Seine)

Mme Belaisch-Allart (1,2,3), M. Vogt (4)

- Centre hospitalier de Courbevoie (Hauts de Seine)

Mme Dolley, MM. Barbot et Stanovici $(1,2)$

- Clinique des Noriets, Vitry sur Seine (Val de Marne)

MM. Larne et Quantin (1,2)

- Clinique du Parisis, Cormeilles en Parisis (Val d'Oise)

M. Boulanger $(1,2)$

REGION LANGUEDOC-ROUSSILLON

- CHRU de Montpellier

M. Hédon $(1,2,3,4)$

- Clinique Saint Pierre, Perpignan

MM. Valentin et Lafont $(1,2)$

- CHRU de Nimes

Mmes Taillant, Ripart-Neveu et M. Mares (1,2), MM. Costa et Navratil (4)

- Clinique Saint Roch, Montpellier

MM. Carbasse, Lelaidier et Mme Rouard-Tourne $(1,2)$

\section{REGION LIMOUSIN}

- CHRU de Limoges

M. Piver $(1,2)$ et M. Dumas (4) 


\section{REGION LORRAINE}

- Maternité Hôpital Ste Croix, Metz

MM. Ragage et Cassier $(1,2,3)$, M. Bouschbacher $(1,2,3,4)$

- CHR de Metz-Thionville, Metz

MM. Delepaul et Girod (4)

- Clinique La Roseraie, Epinal

M. Rento $(1,2)$

- Polyclinique Majorelle, Nancy

M. Cledat $(1,2,4)$

- Maternité Adolphe-Pinard, Nancy

Mmes Guillet-May, Zaccabri et Barbarino-Monniser (1,2,3), M. Hubert (4)

\section{REGION MIDI-PYRENEES}

- Nouvelle clinique de l'Union et du Vaurais, Saint Jean

M. Favrin $(1,2)$

\section{- CHU de Toulouse, Hôpital Lagrave}

M. Monrozies $(1,2,3)$

\section{- CHU de Toulouse, Hôpital Rangueil}

MM. Pontonnier et Soulier (4)

- Clinique Saint Jean, Toulouse

MM. Degoy $(1,2)$ et Gautier (4)

\section{REGION NORD - PAS DE CALAIS}

- CHU de Lille, Hôpital Jeanne de Flandre

M. Leroy et Mmes Billiard-Leroy, Desrousseaux-Thomas et Lefebvre-Maunoury $(1,2,3)$, MM. Rigot et Defossez (4)

- Clinique du Bois, Lille

M. Nguyen-Trong $(1,2)$

- Polyclinique du Parc, Saint Saulve

MM. Morizot et Vanhove $(1,2)$

\section{BASSE-NORMANDIE}

- CHU de Caen

MM. Barjot, Herlicoviez et Von Theobald $(1,2)$

\section{REGION HAUTE-NORMANDIE}

- CHU de Rouen, Hôpital Charles Nicolle

MM. Sibert et Grise (4), Mme Clavier et M. Marpeau $(1,2)$

- Clinique Saint Antoine, Bois Guillaumme

Mme Avril et M. Villiere (1,2), M. Moussu (4)

- CHR du Havre

M. Schweitzer (1,2), M. Villez (4)

\section{REGION PAYS DE LOIRE}

- CHU d'Angers

M. Denis $(1,2)$ 
- Clinique du Tertre rouge, Le Mans

MM. Brière et Mouchel $(1,2)$, M. Poizat $(1,2,4)$

- CHU de Nantes

M. Lopes $(1,2,3)$

- Polyclinique de l'Atlantique, Saint Herblain

MM. Ripoll et Cousin $(1,2)$

- Clinique Notre Dame de Grâces, Nantes

MM. Pousset et Alphandari, Mme Marchand $(1,2)$

- Clinique Saint François, Nantes

M. Le Coguic (4)

\section{REGION PICARDIE}

- CHU d'Amiens

M. Camier (1,2,3), M. Petit (4)

- Clinique Ste Thérèse de l'Enfant Jésus, Amiens

M. Hourdin $(1,2)$, M. Ballot $(1,2,4)$

\section{REGION POITOU-CHARENTES}

- CHU de Poitiers

M. Deshayes $(1,2,3)$

\section{REGION PROVENCE-ALPES-COTE D'AZUR}

- Polyclinique Urbain V, Avignon

MM. Galand et Duvernois $(1,2)$

- Clinique Saint Michel, Toulon

MM. Boschet et Cluzel (1)

- CHU de Marseille, Hôpital La Conception

Mme Galice-Melone (1,2,3), M. Serment (4)

- CHU de Marseille, Hôpital Belle de Mai

M. Gamerre et Mme Noizet $(1,2)$

- Fondation Hôpital Saint Joseph, Marseille

MM. Tourame, Martino et Pascal (1,2), M. Albert (4)

- Clinique Wulfran-Puget, Marseille

MM. Franquebalme, Chincholle et Khouzami (1,2), M. Hermanovicz (4)

- Clinique Saint George, Nice

MM. Barbeault et Nahminovici $(1,2,4)$, M. Charpentier $(1,2)$

- CHU de Nice

M. Gillet et Mme Isnard (1,2,3), M. Bongain (1,2), MM. Chevalier et Toubol (4)

\section{REGION RHONE ALPES}

- CHR de Roanne

MM. Blache et Lemonon (1,2), Mme Mannier (2)

- CHU de Grenoble, Hôpital La Tronche

M. Racinet et Mme Villar (1,2), Mme Grefenstette (2), M. Rambeau (4)

- Clinique Belledonne, Saint Martin d'Hères

MM. Allouch, Benbassa, Muet, Reynes, Sage et Mme Bellaiche (1,2), M. Monod (4) 
- Polyclinique Notre-Dame Auxilliatrice, Valence

Mme Grelat (1,2), M. Allegre (4)

- Clinique Sainte Anne-Lumière, Lyon

MM. Watrelot $(1,2,4)$ et Dreyfus $(1,2)$

- Clinique Monplaisir, Lyon

MM. Bernard, Cohen et Mme Payan (1,2), MM. Dray, Nachury et Cognat $(1,2,4)$

- CHU de Lyon, Hôpital E.Herriot

Mme Boulieu $(1,2,3)$

- CHU de Lyon, Hôpital de la Croix-Rousse

M. Salle $(1,2,4)$

- Polyclinique Sainte Marie-Thérèse, Bron

M.Nicollet (1,2,4) et Mme Chomier (1,2)

- Clinique Michelet, Saint Etienne

M. Griot et Mme Sorg $(1,2)$

- CHU de Saint Etienne, Hôpital Nord

M. Tostain (4)

\section{DEPARTEMENTS D'OUTRE MER}

- Clinique Jeanne d'Arc, Le Port (Réunion)

MM. Baroche et Vicq $(1,2)$

- Centre Hospitalier de Saint-Pierre le Tampon (Réunion)

Mme Gabrielle (1,2)

- CHR de Cayenne (Guyanne)

MM. Stien et Patient $(1,2)$

- Clinique Sainte Marie, Schoelcher (Martinique)

M. Quist $(1,2)$

- Clinique Les Rosiers, Providence aux Abymes (Guadeloupe)

MM. Bangou, Peretti et Mme Marie $(1,2)$

\section{Activités biologiques}

1c - Recueil et traitement du sperme en intraconjugal.

1d - Recueil et traitement du sperme avec tiers donneur.

$2 c$ - Traitement des ovocytes en intraconjugal.

$2 d$ - Traitement des ovocytes avec tiers donneur.

3 - Fécondation in vitro sans micromanipulation.

4 - Fécondation in vitro avec micromanipulation.

$5 c$ - Conservation des gamètes en intraconjugal.

$5 \mathrm{~d}$ - Conservation des gamètes avec tiers donneur.

6c - Conservation des embryons en intraconjugal.

6d - Conservation des embryons avec tiers donneur.

7 - Ensemble des activités biologiques. 


\section{REGION ALSACE}

- CHU de Strasbourg :

Mme Wittemer et M. Gerlinger (1c, 2cd, 3, 4, 6cd), Mme Cranz et M. Clavert (1cd, 2cd, 5cd, 6cd).

- CHR de Mulhouse :

Mme Schmitt (1c, 5cd).

- LABM Klumpp, Strasbourg :

MM. Klumpp et Marx (1c).

- LABM Lang, Strasbourg :

M. Lang (1c).

- LABM Barraud, Colmar :

MM. Barraud et Neuman (1c).

- LABM du Diaconat, Mulhouse :

M. Pierrot (1c).

- LABM du Bollwerk, Mulhouse :

M. Gathrat (1c).

- LABM du Vignoble, Kayserberg :

M. Hachette (1c).

- LABM Monnier, Cernay :

Mme Monnier (1c).

- LABM Pegon, Mulhouse :

Mme Pegon (1c).

\section{REGION AQUITAINE}

- CHU de Bordeaux :

M. Mayer (7), Mme Mathieu (1c, 2cd, 3, 4, 5c, 6c), Mme Papaxanthos (1cd, 2cd, 3, 4), M. Berjon (1cd, 5cd, 6cd).

- CHR de Périgueux :

Mme Dirat (1c).

- LABM Centre de biologie med., Périgueux :

M. Amouroux (1c, 2cd, 5c, 6cd).

- LABM Ruffié, Bordeaux :

M. Seroussi (1c, 2c, 3, 4, 5c, 6c).

- LABM Clavère, Bayonne :

M. Clavère et Mme Cous (1c, 2c, 3, 5c, 6c).

- LABM Etcharry, Pau :

Mme Grabost et M. Uthurriague (1c, 2c, 3, 5c, 6c).

- LABM de Langon :

Mme Bouin (1c). 
- CHU de Clermont Ferrand :

REGION AUVERGNE

MM. Boucher et Jany (1cd, 2cd, 3, 5cd, 6cd), Mme Grizard (1cd, 5cd, 6cd), M. Jimenez (2cd, 3, 4).

\section{REGION BOURGOGNE}

- CHU de Dijon :

M. Zahnd (1cd, 2cd, 3, 5cd, 6cd).

- LABM Bourgeon, Dijon :

Mme Laurin (1c, 2c, 3, 5c).

- Lab. régional de biol. méd., Dijon :

M. Curie (1c, 2cd, 3, 5c, 6cd).

- LABM Centre Labo., Chalon sur Saone :

Mmes Barba et Pomel (1c).

- LABM Dehenry-Melin, Sens

MM. Dehenry et Melin (1c)

\section{REGION BRETAGNE}

- CHU de Brest :

M. Amice (1c, 2cd, 3, 5c, 6cd), Mme Amice (1c,2cd,3,4,5c,6cd)

- CHU de Rennes :

MM. Le Lannou et Griveau (7).

- LABM L'Emeillat, Brest :

M. L'Emeillat (1c, 2cd, 3, 5c, 6cd), M. Velez de la Calle (1c, 2cd, 3, 4, 5c, 6cd).

- LABM Richier, Rennes :

M. Colleu (1c, 2cd, 3, 4, 5c, 6cd).

- LABM Scp Morice, Lorient :

M. Morice (1c), M. Vallée (1c, 2c, 3, 6cd).

- LABM Leclercq, Lorient :

MM. Marot et Leclercq (1c).

- LABM Rebour, Lannion :

M. Rebour (1c).

- LABM des Douves, Quimper :

M. Becam (1c).

- LABM Glasgow, Brest :

MM. Prigent et Scavinner (1c).

- LABM Niaussat, Vannes :

MM. Brault et Niaussat (1c).

- LABM Subileau-Fontaine-James, Ploemerl

M. James (1c) 


\section{REGION CENTRE}

- CHU de Tours :

M. Royère (7), M. Hamamah (1cd, 2cd, 3, 4, 6cd), Mme Barthélémy (1cd, 5cd).

- LABM Arnaud, Tours :

M. Dudragne (1c, 2cd, 3, 5c, 6cd).

- LABM Métadier, Tours :

M. Painsonneau (1c, 2cd, 3, 5c, 6cd).

- LABM Juillard, Orléans :

M. Medallel (1c).

- CHG de Chartres :

MM. Foucault et Laudot et Mme Bouret (1c).

\section{REGION CHAMPAGNE-ARDENNES}

- CHU de Reims :

Mme Pigeon (7), Mme Melin-Blocquaux (1cd, 2cd, 3, 5cd, 6cd), M. Adnet (1d, 5d).

- LABM Leulier, Reims :

M. J. Leulier (1c, 2cd, 3, 5c, 6cd), M. P. Leulier (1c, 2cd, 3, 4, 5c, 6cd).

- CH de Vitry le François :

M. Matazaud (1cd).

- LABM Plas Mollet, Troyes :

M. Mollet (1c).

- LABM Garnier, Reims :

M. Nowak (1c).

- LABM Gillard, Reims :

M. Gillard (1c).

\section{REGION FRANCHE COMTE}

- CHU de Besançon :

M. Bresson (1cd, 2cd, 3, 5cd, 6cd), M. Roux (1c, 2cd, 3, 4, 5c, 6c), Mme Clavequin (1cd, 5cd, $6 \mathrm{~cd}$ ).

- LABM Museur, Besançon :

M. Museur (1c, 2cd, 3, 5c, 6cd), M. Bertrand (1c, 2cd, 3, 4, 5c, 6cd).

\section{REGION ILE DE FRANCE}

- CHU, Hôpital Bichat, Paris 18ème :

Mme Devaux (1cd, 2cd, 3, 6cd).

- CHU, Hôpital A. Béclère, Clamart (Hauts de Seine) :

Mmes Selvat et Bergère (7).

- CHU, Hôpital Jean Verdier, Bondy (Seine St Denis) :

Mme Martin-Pont (1cd, 2cd, 3, 4, 5cd). 
- CHU, Hôpital Cochin, Paris 14ème :

M. Jouannet (7), M. Wolff (1cd, 2cd, 3, 4), Mme Czyglik et MM. Kunstmann et Auger

(1cd, 5cd, 6cd), Mme Poirot (1cd, 2cd, 3, 4, 6cd), M. Lucas (1cd, 2cd, 3)

- CHU, Hôpital Necker, Paris 15ème :

Mmes Kutenn, Alnot, Letur-Kornisch (1cd, 2d, 5cd, 6cd). Mmes Mandelbaum et

Plachot (7).

- CHU, Hôpital Tenon, Paris 20 ème :

MM. Vendrely et North (1cd, 5cd, 6cd), Mme Straub (1c), Mme Mandelbaum (1c, 2cd, 3, 4, 5cd, 6cd).

- CHU, Hôpital La Pitié Salpétrière, Paris 13ème :

M. Gonzales et Mme Lesourd (1c, 2cd, 3, 4, 6cd).

- CHU, Hôpital Bicêtre, Le Kremlin-Bicêtre (Val de Marne) :

M. Soufir (1c, 5c).

- CHU, Hôpital A. Paré, Boulogne (Hauts de Seine) :

Mmes Albert et Guillot et M. Roussel (1cd).

- CHU, Hôpital St Antoine, Paris 12ème :

Mmes Marmor et Schammaneche (1c).

- CH intercommunal de Poissy (Yvelines) :

M. Bisson (1cd, 2cd, 3, 5c, 6cd), Mme Merlet (1cd, 2cd, 3, 4, 5c, 6cd).

- CH intercommunal J. Rostand, Sèvres (Hauts de Seine) :

Mme Plachot (7).

- Hôpital des Diaconesses, Paris 12ème :

Mme Skiada (1c, 2c, 3, 6c).

- Institut mutualiste Montsouris, Paris 14ème :

M. Granet (1c, 2cd, 3, 4, 5c, 6cd).

- CH de Courbevoie (Hauts de Seine) :

Mmes Bonnaud et Guibert (1c, 2c, 3, 6cd).

- LABM de l'Hôpital Américain de Paris, Neuilly (Hauts de Seine) :

MM. Testart et Briot (1c, 2c, 3, 4, 5c, 6cd).

- LABM d'Eylau, Paris 16ème :

MM. Cohen Bacrie et Le Meur, Mmes Dumont et Junca (1c, 2c, 3, 4, 5c, 6cd).

- LABM de la Clinique d'Aubervilliers (Seine St Denis) :

MM. Briot et Malafosse (1c, 2c, 3, 4, 6cd).

- LABM Zerah, Bagnolet (Seine St Denis) :

Mme Zerah et M. Taar (1c, 2c, 3, 4, 5c, 6cd).

- LABM Kabla, Cormeilles (Val d'Oise) :

M. Kabla (1c, 2c, 3, 4, 6cd).

- LABM Millet, Vitry sur Seine (Val de Marne) :

Mmes Millet et Marotte (1c, 2c, 3, 6cd).

- LABM Cabrol, Le Chesnay (Yvelines) :

M. Dugas (1c, 2c, 3, 4, 6cd). 
- LABM de Clamart (Hauts de Seine) :

M. Benhaim (1c).

- LABM Velpeau, Antony (Hauts de Seine) :

MM. Sustra et Languet (1c).

- LABM Suchet, Paris 19ème :

M. Lupu (1c).

- LABM de la Scala, Paris 15ème :

M. Jondet (1c).

- LABM Denis, Paris 14ème :

Mme Denis (1c).

- LABM Drouot, Paris 9ème :

M. Cassuto (1c, 2c, 3, 4, 5c, 6cd)).

- LABM Bijaoui, Paris 6ème :

M. Bijaoui (1c).

- LABM Magenta, Paris 10ème :

Mme Askienazy-Elbar (1c).

- LABM Olivero, Paris :

Mmes Lablache et Bailly, M. Cohen (1c).

- LABM Chaste, Torcy (Seine et Marne) :

M. Rostoker (1c).

- LABM du Vert Galant, Tremblay (Seine St Denis) :

M. Sasporte (1c).

- LABM Perrault, Enghien les Bains (Val d'Oise) :

Mme Perrault (1c).

- LABM Rouge, Champigny sur Marne (Val de Marne) :

M. Rouge (1c).

- LABM du centre Créteil Soleil, Créteil (Val de Marne) :

MM. Rambert et Monard (1c).

- LABM Mintz, Aubergenville (Yvelines) :

Mme Mintz (1c).

- LABM Clement, Le Blanc Mesnil (Seine St Denis)

M. Clement (1c, 2c, 3, 5c, 6cd)

\section{REGION LANGUEDOC ROUSSILLON}

- CHU de Nîmes :

M. Archambaut (1cd, 2c, 3, 4, 5cd, 6cd).

- CHU de Montpellier :

M. Humeau (1cd, 2cd, 3, 5cd, 6cd), Mme Arnal (1cd, 2cd, 3, 4, 5c, 6cd), M. Chalet (1cd, 5cd).

- LABM Soulié, Nîmes :

M. Soulié (1c, 2c, 3, 5c, 6cd), M. Regnier-Vigouroux (1c, 2c, 3, 4, 5c, 5cd) 
- Centre de Biologie, Narbonne :

M. Forestier (1c).

- SCP Bluche, Carcassone :

Mme Bluche-Guilhem (1c).

- LABM de la Cité, Carcassonne

M. Zeglany (1c)

- LABM Bovidan, Alès :

Mme Bovidan-Vivinie (1c).

- LABM Fons, Mende :

M. Fons (1c)

- LABM Fourquet, Nîmes :

Mme Fourquet-Canonne (1c).

- LABM Giovanetti, Bagnols sur Cèze (Gard) :

Mmes Giovanetti et Pratlong (1c).

- SCP Bonneton, Montpellier :

Mme Bonneton (1c).

- LABM Illes, Montpellier :

M. Illes (1c).

- LABM Réal, Béziers :

M. Réal (1c).

- Centre de Biologie Médicale, Perpignan :

M. Abecassis (1c).

- Laboratoire central de Biologie, Perpignan :

Mme Raynaud (1c).

\section{REGION LIMOUSIN}

- CHR Dupuytren, Limoges :

Mme Paulhac (1cd, 2c, 3, 5cd, 6cd)

- LABM Castro, Limoges :

M. Castro (1c)

\section{REGION LORRAINE}

- CHU, Maternité Pinard, Nancy :

M. Foliguet (7), M. Gérard (1cd, 2cd, 3, 4, 6cd), Mme Touati (1cd, 5cd, 6cd).

- LABM Stahl, Metz :

M. Wasels (1c, 2c, 3, 4, 5c, 6cd), M. Kuntzel (1c, 2c, 3, 5c, 6cd).

- LABM Gonand, Epinal :

M. Lefaure (1c, 2c, 3, 6cd), M. Dieteman (1c).

- LABM Cinqualbre, Nancy :

M. Paulus (1c, 2c, 3). 
- CHR de Metz-Thionville, Metz :

MM. Stoessel et Pax, Mme Portenseigne (1c).

- LABM Brignon, Nancy :

MM. Baillet et Taboul (1c).

- LABM Dory, Sarreguemines :

M. Dory (1c).

- LABM Pax, Metz :

M. $\operatorname{Pax}(1 \mathrm{c})$.

\section{REGION MIDI-PYRENEES}

- CHU, Hôpital La Grave, Toulouse :

Mme Mansat et M. Bujan (1cd, 5cd, 6cd), M. Parinaud (7).

- LABM Montagut, Toulouse :

M. Montagut (1c, 2cd, 3, 4, 5c, 6cd).

- IFREARES, Toulouse :

M. Montagut (1d, 5d), M. Favrin (recueil d'ovocytes en vue de don).

- CHR de Foix :

Mme Clarac (1c).

- LABM Bensaïd, Rodez :

M. Bensaïd (1c).

- LABM Fleurquin, Rodez :

M. Bouilloux (1c).

- LABM Cros, Millau

Mme Cros (1c)

\section{REGION NORD-PAS DE CALAIS}

- CHU de Lille :

M. Hermand (1cd, 2cd, 3, 5cd, 6cd), M. Defossez (1cd,2cd,3) M. Soulez (1cd, 2cd, 3, 4), Mmes Leroy-Martin et Saint-Pol et M. Leonardelli (1cd, 5cd, 6cd).

- SCP Leduc, Lille :

M. Fourlinnie et Mme Couplet (1c), Mme Louvet (2c, 3, 5c, 6cd), M. Herbaut (2c, 3, 4, 5c, 6cd).

- LABM Rouimini, Roeulx :

M. Rouimini (1c, 2c, 3, 6cd).

- LABM Liberté, Lille :

MM. Lesur et Dieusaert (1c, 2c, 3, 6cd).

- LABM Devilliers, Bethune :

M. Devilliers (1c)

- LABM Lecerf, Dunkerque :

MM. Ficheux et Callens (1c).

- LABM Momal, Valenciennes :

MM. Demaeght et Gadeyne (1c, 2c, 3, 4, 5c, 6cd). 
- Centre de Biologie du Calaisis, Calais :

MM. Andlauer et Gaeremynck (1c).

- LABM Demarquilly, Henin-Beaumont :

M. Canonne (1c).

- LABM Sagot, Boulogne sur Mer :

M. Sagot (1c).

- LABM Schaffner, Lens

Mme Wallon, MM. Schaffner et Bart (1c)

\section{REGION BASSE NORMANDIE}

- CHU de Caen :

Mme Sauvalle (1cd, 5cd, 6cd), Mme Denis (7), Mme Herlicoviez (1cd, 2cd, 3, 4, 5cd).

- CHR d'Alençon :

M. Marie (1c).

- LABM Saint Martin, Caen :

Mme Chocat (1c).

- LABM Visseaux, Lisieux :

Mme Visseaux (1c).

- LABM Launay, Cherbourg :

M. Queffeulou (1c).

- LABM Saudin, Cherboug :

M. Saudin (1c)

- LABM Campet, Argenton :

M. Eugène (1c).

\section{REGION HAUTE NORMANDIE}

- CHU de Rouen :

M. Macé et Mme Rives (1cd, 2cd, 3, 5cd, 6cd).

- Centre de biologie médicale, Le Havre :

M. Rocaboy (1c, 2c, 3, 4, 5c, 6cd).

- LABM Prentout, Rouen :

M. Bastit (1c, 2c, 3, 4, 5c, 6cd), M. Gray (1c, 2c, 3, 5c, 6cd).

\section{REGION PAYS DE LOIRE}

- CHU de Nantes :

M. Barrière (1cd, 2c, 3, 4, 5cd, 6cd).

- CHU d'Angers (Maine et Loire) :

Mme Chrétien (1c, 2c, 3).

- LABM Trichereau, Nantes :

M. Plouchart (1c, 2c, 3, 4, 6cd). 
- LABM Faucheus, Le Mans :

MM. Joly et Sigogneau (1c, 2c, 3, 4, 5c, 6cd).

- CHR du Mans :

M. Marie (1c).

- LABM Fonty, Angers :

M. Benon (1c).

- LABM Friteau, Laval :

M. Roche (1c).

\section{REGION PICARDIE}

- CHU d'Amiens :

M. Thépot (7).

- LABM Albert 1er :

M. Bourdrel et Prévot (1c, 2c, 3, 4, 5c, 6cd).

- CH de Creil :

M. Lemaitre (1c).

- CH de Beauvais :

Mme Heurte (1c).

- LABM Laurian, Compiègne :

M. Ciolkovitch (1c).

\section{REGION POITOU-CHARENTES}

- CHU de Poitiers :

M. Chansigaud (1c, 2cd, 3, 5c, 6cd).

- LABM du Château, Angoulême :

MM. Leix-Cote et Labrousse (1c).

REGION PROVENCE-ALPES-COTE D'AZUR

- CHU, Hôpital Belle de Mai, Marseille :

M. Grillo (1cd, 2cd, 3, 4).

- CHU, Hôpital La Conception, Marseille :

M. Luciani (7), Mme Magnon (1cd, 2cd, 3, 4, 6cd).

- CHU, Hôpital Pasteur, Nice :

Mme Donzeau (1cd, 2c, 3, 4, 5cd, 6cd), M. Fenichel (1cd, 5cd, 6cd).

- LABM Chaudon-Daumas, Nice :

Mme Richelme-Duforestel (1c, 2c, 3, 5c, 6cd), M.Pierre (1c)

- LABM Soubiran, Nice :

Mme Delpech (1c, 2, 3), M. Soubiran (1c).

- LABM Hôpital St Joseph, Marseille :

M. Boyer (1cd, 2cd, 3, 4, 6cd). 
- LABM Caparros, Marseille :

MM. Giorgetti et Terrion (1c, 2c, 3, 4, 5c, 6cd).

- LABM Hubert Lumbroso, Marseille :

M. Celse-L'Hoste et Mme Frison-Peretti (1c, 2c, 3, 6cd).

- LABM Orfanos, Avignon :

MM. Gras et Roudon (1c, 2c, 3, 6cd).

- Institut de Médecine de la Reproduction, Marseille :

M. Roulier (1d, 5d).

- LABM Drai-Obadia, Marseille :

Mme Drai-Obadia (1c).

- LABM Bourgoin, Toulon

Mme Cohen-Billiemaz (1c)

- LABM Combes, Toulon

Mme Combes (1c)

- CHR de Draguignan :

Mme Lanoe-Joly (1c).

- LABM Demes, Cannes :

M. Demes (1c).

- LABM Eynaud-Bo, Marseille :

Mlle Bray (1c).

- LABM Girodengo, Aix en Provence :

Mme Girodengo (1c).

- LABM Grellet, Aix en Provence

M. Grellet (1c).

- LABM Martin, Bollène :

Mme Martin (1c).

- CHR D'Ajaccio

M. Valayer (1c)

\section{REGION RHONE-ALPES}

- CHU de Saint Etienne :

M. Maubon (1c, 2c, 3, 5c, 6cd).

- CHR de Roanne :

M. Szymanovicz et Mme Neyron (1c, 2c, 3).

- CHU de Grenoble :

M. Jalbert et Mme Servoz-Gavin ( $1 \mathrm{~cd}, 5 \mathrm{~cd}, 6 \mathrm{~cd})$, M. Sèle et Mmes Jacob-Bergues et Rousseaux (1c, 2cd, 3, 4, 5c, 6cd).

- CHU de Lyon, Hôpital Edouard Herriot :

M. Guérin et Mme Lornage (1c, 2cd, 3, 4), Mme Cottinet (1d, 5cd, 6cd). 
- LABM De Clercq, Valence :

M. De Clercq (1c, 2cd, 3, 4, 6cd), M. Lavaud (1c, 2cd, 3, 6cd).

- LABM Chouteau, Saint Egrève :

M. Chouteau (1c, 2cd, 3, 4, 5c, 6cd).

- LABM Bouquet, Villeurbanne :

M. Defretin (1c, 2cd, 3, 4, 5c, 6cd).

- LABM Merieux, Lyon :

M. Menezo (1c, 2cd, 3, 4, 5c, 6cd).

- LABM Vincent, Lyon :

Mme Pilikian (1c, 2c, 3, 4).

- LABM Bretin, Bourg en Bresse :

Mme Pernoud et Mr Megevand (1c).

- LABM d'Annecy :

M. Martin (1c).

- LABM Mendez, Annecy :

Mme Mendez (1c).

- LABM Lacassagne, Lyon :

M. Molgatini (1c).

- LABM Mazuyer, Lyon :

M. Mazuyer (1c).

- LABM Ingels, Villefranche S/Saône :

Mmes Vignon et Peyle (1c)

- LABM Tercinet, Chambéry :

M. Boulanger (1c)

- LABM Berlioz, L'Isle d'Abeau :

M. Berlioz (1c)

DEPARTEMENTS D'OUTRE-MER

- LABM Espiand-Girard, Pointe à Pitre (Guadeloupe) :

Mme Espiand-Girard (1c, 2c, 3, 5c, 6cd).

- LABM Plenet, Cayenne (Guyane) :

MM. Plenet et Bonnevie (1c, 2c, 3).

- LABM Audenay, Schoelcher (Martinique) :

M. Audenay (1c, 2c, 3, 5c, 6cd).

- LABM Verroughstraete, Le Port (Réunion) :

M. Verroughstraete et Mme Gayon (1c, 2c, 3, 4, 5c, 6cd). 